\title{
COMPACTNESS PROPERTIES OF TOPOLOGICAL GROUPS
}

\author{
BY \\ S. P. WANG( ${ }^{(1)}$
}

\begin{abstract}
In a paper of R. Baer and later in a paper of B. H. Neumann, finiteness properties of groups have been studied. In the present paper, we develop their analogous notions in topological groups and even sharpen some of their results.
\end{abstract}

In [1] R. Baer has studied the finiteness properties of groups and in [8] B. H. Neumann has studied groups with finite classes of conjugate elements. It is the aim of this paper to study the analogous notions in topological groups. We have generalized and sharpened some of the results in [1], [8], [10]. Some structure theorems of topological groups are presented.

Notations. Let $G$ be a topological group. An element $x$ of $G$ is called periodic if $x$ is contained in a compact subgroup of $G$. We denote by $P(G)$ the set of all periodic elements of $G$. An element $x$ of $G$ is called conjugate-bounded if the

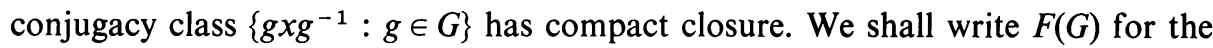
set of all conjugate-bounded elements of $G$. Clearly $F(G)$ is a characteristic subgroup of $G$ and in general $P(G)$ is not a subgroup of $G$. A topological group is called a $F C$-group if $G=F(G)$. Given subsets $X$ and $Y$, we write $C(X, Y)$ for the subset $\left\{x y x^{-1}: x \in X, y \in Y\right\}$; in particular for any $x \in G, C(G,\{x\})$ is just the conjugacy class containing $x$.

1. Groups with compact-open subgroups. In this section, we shall establish some properties of topological groups with compact-open subgroups. Let $G$ be a topological group and $K$ be a compact-open subgroup of $G$.

THEOREM 1.1. Let $G, K$ be described as above and $A$ be a subset of $P(G)$ with compact closure. If $A$ satisfies the conditions $e \in C(A, A) \subset A=A^{-1}$ and $A K=K A$, then we have the following:

(i) There is a finite subset $\left\{a_{1}, \ldots, a_{n}\right\}$ of $A$ with

$$
\left\{a_{1}, \ldots, a_{n}\right\} K=K\left\{a_{1}, \ldots, a_{n}\right\}=A K=K A .
$$

(ii) There exists a positive integer $m$ with $a_{i}^{m} \in K(i=1, \ldots, n)$.

Received by the editors February 10, 1970.

AMS 1969 subject classifications. Primary 2210, 2220.

Key words and phrases. Periodic elements, FC-groups, Z-groups, Lie groups, quasicompact, algebraic groups.

(1) Partially supported by NSF-GP-8499. 
(iii) $A^{s} K$ is a group, for $s \geqq n(m-1)$; in particular the subgroup generated by $A$ has compact closure.

Proof. (i) Since $K$ is an open subgroup, $A K$ is both open and closed. Hence $A K=\bar{A} K$ is compact and (i) follows easily.

(ii) Since $\left\{a_{1}, \ldots, a_{n}\right\}$ is contained in $P(G)$ and $K$ is an open subgroup of $G$, the existence of such positive integer $m$ is immediate.

(iii) By assumption, $e \in A=A^{-1}$; hence the subgroup $\langle A\rangle$ generated by $A$ is the union of the subsets $A^{s}(s=1,2, \ldots)$. Clearly by assumption, $(A K)^{-1}=K^{-1} A^{-1}=$ $K A=A K$ and the group $\langle A K\rangle$ generated by $A K$ is $\langle A\rangle K$. Hence it suffices to verify $\langle A\rangle \subset A^{s} K$ for $s \geqq n(m-1)$. Let $x$ be any element of $\langle A\rangle$ and $\lambda$ be the smallest positive integer with $x \in A^{\lambda} K$. Suppose that $\lambda>n(m-1)$. From (i), $A^{\lambda} K=\left\{a_{1}, \ldots, a_{n}\right\}^{\lambda} K$ and thus $x$ has the expression

$$
x=b_{1} \cdots b_{\wedge} k,
$$

where $k \in K$ and $b_{j} \in\left\{a_{1}, \ldots, a_{n}\right\}(1 \leqq j \leqq \lambda)$. As $\lambda \geqq 1+n(m-1)$, there is one index $i$ such that $a_{i}$ appears among $b_{1}, \ldots, b_{\lambda}$ at least $m$ times. For $C(A, A) \subset A$, by bringing the term $a_{i}$ together in (1.2) through conjugation, we obtain

$$
x=b_{1} \cdots b_{t-1} a_{i}^{m} c_{t} \cdots c_{\lambda-m} k
$$

where $c_{j} \in A(t \leqq j \leqq \lambda-m)$. By our choice of $m, a_{i}^{m} \in K$. Since $A K=K A$, we can rewrite (1.3) in the form

$$
x=b_{1} \cdots b_{t-1} d_{t} \cdots d_{\lambda-m} k^{\prime}
$$

where $k^{\prime} \in K$ and $d_{j} \in A(t \leqq j \leqq \lambda-m)$. Hence $x \in A^{\lambda-m} K$ which contradicts our choice of $\lambda$. Therefore $\lambda \leqq n(m-1)$ and $\langle A\rangle \subset A^{s} K$ for $s \geqq n(m-1)$. Now the other assertion follows easily and the proof is completed.

As an immediate consequence of the theorem, we have the following corollary due to Ušakov.

COROLlARY 1.5 (UŠAKOV [10]). Let $G$ be a topological group and $B$ a normal subset of $P(G)$ with compact closure. If $G$ has a compact-open subgroup, then the group $\langle B\rangle$ generated by $B$ is a normal subgroup of $G$ with compact closure.

Proof. Let $A=B \cup B^{-1} \cup\{e\}$. Clearly $A$ satisfies all the conditions of the preceding theorem. By (iii), $\langle A\rangle=\langle B\rangle$ has compact closure.

REMARK. As we shall prove later, the assumption of the existence of compactopen subgroups in the preceding corollary can be dropped for locally compact groups. However the general case depends heavily on this special case and the structure of $F C$-groups.

Corollary 1.6 [8]. Let $G$ be a group and $Z(G)$ its center. If the index $[G: Z(G)]$ is finite, then $[G, G]$ is finite. 
Proof. Let $D$ be a divisible abelian group containing $Z(G)$ and let the following diagram

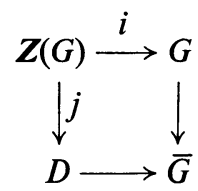

be the push-out of the maps $i, j$, where $i, j$ are the inclusion maps of $Z(G)$ into $G$ and $D$ respectively. Clearly $D$ is central in $\bar{G}$ and divisible, and $\bar{G} / D$ is isomorphic to $G / Z(G)$. Hence there are periodic elements $x_{1}, \ldots, x_{n}$ in $\bar{G}$ with $\bar{G}=D \cdot\left\langle x_{1}, \ldots, x_{n}\right\rangle$. With respect to discrete topology, $\bar{G}$ is an $F C$-group. Thus by Corollary 1.5 , the union of the conjugacy classes $C\left(\bar{G},\left\{x_{i}\right\}\right)(1 \leqq i \leqq n)$ is contained in a finite normal subgroup of $\bar{G}$. Hence $\left\langle x_{1}, \ldots, x_{n}\right\rangle$ is finite. Clearly $\left\langle x_{1}, \ldots, x_{n}\right\rangle$ is a normal subgroup of $\bar{G}$ and $\bar{G} /\left\langle x_{1}, \ldots x_{n}\right\rangle \cong D /\left(D \cap\left\langle x_{1}, \ldots, x_{n}\right\rangle\right)$ is abelian. Therefore $[G, G]$, contained in $\left\langle x_{1}, \ldots, x_{n}\right\rangle$, is finite.

PROPOSITION 1.8. Let $G$ be a topological group with a maximal compact-open subgroup. Then we have the following:

(i) $P(G) \cap F(G)$ is a compact characteristic subgroup of $G$; more precisely $P(G) \cap F(G)$ is the intersection of all maximal compact-open subgroups of $G$.

(ii) $F(G)$ is closed and $F(G) \cap P(G)$ is open in $F(G)$.

(iii) $F(G) / F(G) \cap P(G)$ is a discrete torsion free abelian group.

Proof. Let $K$ be any maximal compact-open subgroup of $G$ and $x \in P(G) \cap F(G)$. By Corollary $1.5, x$ is contained in a compact normal subgroup $N(x)$ of $G$. Clearly $K N(x)$ is a compact-open subgroup of $G$, hence $N(x) \subset K$ and $F(G) \cap P(G) \subset K$. Since $K$ is arbitrary, $F(G) \cap P(G)$ is contained in the intersection $L$ of all maximal compact-open subgroups of $G$. Since $L$ is a compact normal subgroup of $G, L$ is contained in $F(G) \cap P(G)$. Therefore (i) follows.

(ii) Clearly $P(G)$ contains a compact-open subgroup of $G$. $F(G) \cap P(G)$, containing an open subgroup of $F(G)$, is open and closed in $F(G)$. It follows that $F(G) \cap P(G)=\mathrm{Cl}(F(G) \cap P(G))$ is open in $\mathrm{Cl}(F(G))$. Hence one concludes readily $F(G)$ is closed in $G$.

(iii) From (ii), $F(G) \cap P(G)$ is open in $F(G)$. Hence the group $F(G) /(F(G) \cap P(G)$ ) is discrete. Let $\bar{G}=F(G) /(F(G) \cap P(G))$. Clearly $F(\bar{G})=\bar{G}$. Let $x$ be any element of $F(G)$ such that its image $\bar{x}$ in $\bar{G}$ is periodic. One sees easily $x \in P(G)$, hence $\bar{G}$ is torsion free. Let $\alpha, \beta$ be any two elements of $\bar{G}$ and $H$ be the subgroup $\langle\alpha, \beta\rangle$. Since $H$ is discrete and $F(H)=H$, both the centralizers $Z_{H}(\alpha)$ and $Z_{H}(\beta)$ of $\alpha$ and $\beta$ in $H$ respectively are of finite index in $H$. Hence $[H: Z(H)]$ is finite. By Corollary $1.6,[H, H]$ is finite. As $\bar{G}$ is torsion free, $[H, H]=\{e\}$. Since $\alpha, \beta$ are arbitrary, $\bar{G}$ is abelian. Therefore the proof is completed.

The condition in the preceding proposition is rather restricted. However, the following proposition gives us a sufficient condition for a locally compact group to have maximal compact-open subgroups. 
Proposition 1.9. Let $G$ be a topological group with compact-open subgroups. If $G$ has a torsion free discrete subgroup $\Gamma$ such that $G / \Gamma$ is compact, then $G$ has maximal compact-open subgroups.

Proof. Let $\mu$ be a Haar measure of $G$ and $K$ be a compact-open subgroup of $G$. Clearly $\mu(G / \Gamma)$ is finite and $K \cap x \Gamma x^{-1}=\{e\}$ for all $x \in G$. We write $G$ as the disjoint union of the double cosets $K x_{i} \Gamma(i \in I)$. Since $\mu$ is unimodular $\left({ }^{2}\right), \mu(G / \Gamma)$ $=n \cdot \mu(K)$ where $n$ is the cardinal number of the index set $I$. From this $n$ is finite. Let $K^{\prime}$ be any compact subgroup of $G$ containing $K$. Clearly $\mu\left(K^{\prime}\right)=\mu(K) \cdot\left[K^{\prime}: K\right]$ $\leqq \mu(G / \Gamma)=n \cdot \mu(K)$. It follows that $\left[K^{\prime}: K\right] \leqq n$ is bounded; in particular $G$ has maximal compact-open subgroups containing $K$.

Corollary 1.10. Let $G$ and $\Gamma$ satisfy the conditions of Proposition 1.9. Then (i) $F(G) \cap P(G)$ is a compact characteristic subgroup of $G$. (ii) $F(G)$ is closed in $G$. (iii) $F(G) /(F(G) \cap P(G))$ is a discrete $F C$-group $\left({ }^{3}\right)$.

Proof. Immediate from Propositions 1.8 and 1.9.

2. Z-groups. Let $G$ be a locally compact group and $Z(G)$ be its center. $G$ is called a $Z$-group if $G / Z(G)$ is compact. $Z$-groups have been studied very extensively in [4]. Here we shall quote the structure theorem of $Z$-groups without proof.

THEOREM 2.1 [4]. Let $G$ be a Z-group. Then $G$ is the direct product of a vector group $V\left({ }^{4}\right)$ and a locally compact group $H$ having a normal compact-open subgroup.

As the consequence of Theorem 2.1 and Corollary 1.6, we have the following generalization of Corollary 1.6.

COROLlaRY 2.2 Let $G$ be a Z-group. Then $[G, G]$ has compact closure.

In the following, we shall generalize Corollary 2.2 furthermore. The discrete case is due to $\mathrm{R}$. Baer.

THEOREM 2.3. Let $G$ be a locally compact group having compact-open subgroups and $L, M$ be closed normal subgroups of $G$. If the centralizers $Z_{M}(L)$ and $Z_{L}(M)$ of $L, M$ in $M$ and $L$ respectively satisfy the condition that $M / Z_{M}(L)$ and $L / Z_{L}(M)$ are compact, then $[L, M]$ has compact closure.

Proof. We shall prove the theorem in several steps.

(i) Let $A=C(L, M) \cup C(M, L)$. Clearly $e \in A=A^{-1}$ and $A$ is a compact normal subset of $G$.

(ii) Let $K$ be a compact-open subgroup of $G$. Clearly there are finite elements $a_{1}, \ldots, a_{n}$ in $A$ with $\left\{a_{1}, \ldots, a_{n}\right\} K=K\left\{a_{1}, \ldots, a_{n}\right\}=K A=A K$.

(2) See Lemma 5 in [9].

$\left({ }^{3}\right)$ This generalizes the result in [13].

${ }^{(4)} V$ is a vector group if $V$ is isomorphic to $R^{n}, 0 \leqq n<\infty$. 
(iii) Since $K \cap M$ is compact-open in $M$ and $L / Z_{L}(M)$ is compact, there is a compact-open subgroup $K_{1}$ of $K \cap M$ invariant under conjugations by elements of $L$. Clearly $Z_{M}(L)$ is a normal subgroup of $G$ and $\left[M: Z_{M}(L) K_{1}\right]$ is finite. Thus there is a positive integer $m$ with $a_{i}^{m} \in Z_{M}(L) K_{1}(1 \leqq i \leqq n)$.

(iv) $A^{s} K$ is a group for $s \geqq n m$. Clearly $\langle A K\rangle=\langle A\rangle K$, hence it suffices to show that $\langle A\rangle$ is contained in $A^{s} K$ for $s \geqq n m$. Let $x$ be any element of $\langle A\rangle$ and $\lambda$ the smallest positive integer with $x \in A^{\lambda} K$. Assume that $\lambda>n m$. From (ii), $A^{\lambda} K=\left\{a_{1}, \ldots, a_{n}\right\}^{\lambda} K$, hence $x$ has the expression

$$
x=b_{1} \cdots b_{\lambda} k
$$

where $k \in K$ and $b_{j} \in\left\{a_{1}, \ldots, a_{n}\right\}(1 \leqq j \leqq \lambda)$. Since $\lambda>n m$, there is $a_{i}$ such that $a_{i}$ appears among $\left\{b_{1}, \ldots, b_{\lambda}\right\}$ at least $m+1$ times. By bringing the term $a_{i}$ together in (2.4) through conjugation, we have

$$
x=b_{1} \cdots b_{t-1} a_{i}^{m+1} C_{t} \cdots C_{\lambda-m-1} k,
$$

where $C_{j} \in A(t \leqq j \leqq \lambda-m-1)$. By (iii) $a_{i}^{m}=k_{1} z$ with $k_{1} \in K_{1}$ and $z \in Z_{M}(L)$. Write $a_{i}=[u, v]=u v u^{-1} v^{-1}$, or $[v, u]$, with $u \in L$ and $v \in M$. Hence we have in case $a_{i}=[u, v]$,

$$
\begin{aligned}
a_{i}^{m+1} & =[u, v]^{m+1}=\left[[u, v]^{m}, u\right] u[u, v]^{m} v u^{-1} v^{-1} \\
& =\left[k_{1} z, u\right] u^{2}\left[v, u^{-1}\right]^{m-1} u^{-2}\left[u^{2}, v\right] \in K_{1} A^{m} .
\end{aligned}
$$

Clearly if $a_{i}=[v, u], a_{i}^{m+1} \in A^{m} K_{1}$. It follows from (2.5) and (2.6) that $x \in A^{\lambda-1} K$ for $K_{1} \subset K$ and $A K=K A$. Thus we get a contradiction to our choice of $\lambda$. Hence we must have $\lambda \leqq n m$. Therefore $A^{s} K$ is a group for $s \geqq n m$ and one concludes readily that $[L, M]=\langle A\rangle$ has compact closure.

REMARK. When $G$ is a Lie group, Theorem 2.3 can be sharpened to assert that $[L, M]$ is compact $\left({ }^{5}\right)$.

3. Some lemmas on linear Lie groups. Let $\operatorname{GL}(n, C)$ (resp. $\mathrm{gl}(n, \boldsymbol{C})$ ) be the group of all $n \times n$ nonsingular complex matrices (resp. the Lie algebra of all $n \times n$ complex matrices). Clearly $\mathrm{gl}(n, C)$ is the Lie algebra of $\mathrm{GL}(n, C)$ and the exponential map exp: $\mathrm{gl}(n, \boldsymbol{C}) \rightarrow \mathrm{GL}(n, C)$ is just the usual one. Let $\lambda$ be any positive number. We denote by $\mathrm{gl}(n, C ; \lambda)$ the set of all elements $X$ in $\mathrm{gl}(n, C)$ such that the imaginary parts of all the eigenvalues of $X$ lie in the open interval $(-\lambda, \lambda)$. Clearly $\mathrm{gl}(n, \boldsymbol{C} ; \lambda)$ is open in $\mathrm{gl}(n, \boldsymbol{C})$ and its closure is contained in any $\mathrm{gl}(n, C ; \mu)$ with $\lambda<\mu$. We write $\exp _{\lambda}$ for the restriction of exp on $\mathrm{gl}(n, C ; \lambda)$ and $\mathrm{GL}(n, C ; \lambda)$ for $\exp (\mathrm{gl}(n, C ; \lambda))$. The following lemma is due to H.-C. Wang [11].

Lemma 3.1 [11]. Let $X$ and $Y$ be two elements of $\mathrm{gl}(n, C ; \pi)$. Then $X=Y$ if and only if $\exp X=\exp Y$.

As a consequence of Lemma 3.1, we have the following proposition.

(5) See Theorem 3.4 in [12]. 
Proposition 3.2. The maps $\exp _{\lambda}: \mathrm{gl}(n, C ; \lambda) \rightarrow \mathrm{GL}(n, C ; \lambda)$ are diffeomorphisms for $0<\lambda \leqq \pi$.

Proof. Under the assumption $0<\lambda \leqq \pi$, by the preceding lemma $\exp _{\lambda}$ is bijective and $C^{\infty}$-differentiable. It is known [5] that the differential of the map exp at $X$ is given by

$$
(d \exp )_{X}=\left(d L_{\exp X}\right)_{e} \cdot\left(\left(1-e^{\operatorname{ad} X}\right) / \operatorname{ad} X\right)
$$

where $L_{\exp X}$ is the left translation $Y \rightarrow(\exp X) Y, Y \in \mathrm{GL}(n, C)$. Thus by a straightforward calculation one concludes that $(d \exp )_{X}$ is bijective for $X \in \mathrm{gl}(n, C ; \pi)$. It follows then that $\exp _{\lambda}$ is a diffeomorphism for $0<\lambda \leqq \pi$.

Let $G$ be any Lie subgroup of $\operatorname{GL}(n, C)$ and $g$ its Lie algebra. Given any $\lambda>0$ we write $g_{\lambda}, G_{\lambda}$ and $\exp _{\lambda}$ for $\mathrm{g} \cap \mathrm{gl}(n, C ; \lambda)$, $\exp \left(\mathrm{g}_{\lambda}\right)$ and the restriction of exp on $\mathrm{g}_{\lambda}$ respectively. From Proposition 3.2 the maps $\exp _{\lambda}(0<\lambda \leqq \pi)$ are diffeomorphisms.

The following lemma is well known. For completeness, we give a proof.

LeMma 3.3. Let $G$ be a subgroup of $\operatorname{GL}(n, C)$. If the entries of all the elements of $G$ are bounded, then $\bar{G}$ is compact.

Proof. Let $\alpha: \operatorname{GL}(n, C) \rightarrow \mathrm{gl}(n+1, C)$ be the map

$$
\alpha(X)=\left(\begin{array}{cc}
X & 0 \\
0 & \operatorname{det} X^{-1}
\end{array}\right), \quad X \in \operatorname{GL}(n, C) .
$$

Clearly $\alpha$ is an embedding and $\alpha(\operatorname{GL}(n, C))$ is closed in $\mathrm{gl}(n+1, C)$. From the assumption $\alpha(\bar{G})$ is a bounded closed subset of $\mathrm{gl}(n+1, C)$. Hence $\alpha(\bar{G})$ is compact. Since $\alpha$ is an embedding, it follows easily that $\bar{G}$ is compact.

4. FC-groups. Let $G$ be a topological group. $G$ is called an $F C$-group if $G=F(G)$. In this section, we shall determine the structure of $F C$ locally compact groups.

Proposition 4.1. Let $G$ be a Lie subgroup of $\mathrm{GL}(n, C)$ and $\mathrm{Ad}: G \rightarrow$ Aut $(\mathrm{g})$ be the adjoint representation. If $F(G)$ is dense in $G$, then $\operatorname{Ad}(G)$ has compact closure.

Proof. Clearly $\mathrm{g}_{\pi / 2}$ is invariant under $\operatorname{Ad}(G)$ and $G_{\pi / 2}$ is a neighborhood of the identity in $G$. Since $F(G)$ is dense in $G$ there are elements $g_{1}, \ldots, g_{n}$ in $G_{\pi / 2} \cap F(G)$ such that the elements $X_{i}=\log \left(g_{i}\right)(1 \leqq i \leqq n)$ form a basis of $g$ over $R$. It is easy to see that the closure of $\operatorname{GL}(n, C ; \pi / 2)$ is contained in $\operatorname{GL}(n, C ; \lambda)$ with $\lambda>\pi / 2$. Hence it follows that the closure of $G_{\pi / 2}$ in $\operatorname{GL}(n, C)$ is contained in $G_{\pi}$. Since $\exp _{\pi}$ is a diffeomorphism, we have that $\mathrm{Cl}\left(\mathrm{Ad}(G) X_{i}\right)$ is homomorphic to $\mathrm{Cl}\left(C\left(G, g_{i}\right)\right)$ $(1 \leqq i \leqq n)$. By our choice, the latter is compact, hence $\mathrm{Cl}\left(\mathrm{Ad}(G) X_{i}\right)$ is compact. Thus with respect to the basis $\left\{X_{1}, \ldots, X_{n}\right\}$, the entries of all the elements of $\operatorname{Ad}(G)$ are bounded. By Lemma 3.3, Ad $(G)$ has compact closure.

Corollary 4.2. Let $G$ be a linear Lie group with dense $F(G)$. Then $G^{0}$, the identity component of $G$, is the direct product of a compact group $K$ and a vector group $V$; moreover $G^{0} / K$ is central in $G / K$. 
Proof. From Proposition 4.1, $\mathrm{Ad}(G)$ has compact closure. Hence $G$ has arbitrary small invariant neighborhoods of the identity. Therefore $G^{0}$ is the product of a compact group $K$ and a vector group $V$. Let $\bar{G}=G / K$. Clearly we may assume that $\bar{G}^{0}=V$ and $F(\bar{G})$ is dense in $\bar{G}$. Let $x \in F(\bar{G})$ and $c_{x}$ be the map $v \rightarrow x v x^{-1} v^{-1}$, $v \in V . c_{x}$ is a homomorphism and $c_{x}(V)$ is a vector subspace of $V$. Moreover $c_{x}(V) \subset x C\left(\bar{G}, x^{-1}\right)$. Since $x C\left(\bar{G}, x^{-1}\right)$ has compact closure, we conclude that $c_{x}(V)=\{e\}$, i.e., $x$ centralizes $V$. As $F(\bar{G})$ is dense, it follows that $V=G^{0} / K \subset Z(G / K)$.

LEMMA 4.3. Let $G$ be a connected Lie group and $Z(G)$ be its center. If the Lie algebra of $G / Z(G)$ is reductive $\left({ }^{6}\right)$, then $G$ is the product of a connected normal semisimple Lie subgroup $S$ and the radical $R(G)$ of $G$.

Proof. Let $R(G)$ be the radical of $G$ and $S$ a maximal connected semisimple Lie subgroup of $G$. From the assumption, given $r \in R(G)$, the map $c_{r}: s \rightarrow r s r^{-1} s^{-1}$, $s \in S$, is a homomorphism from $S$ into $Z(G)$. Since $S$ is semisimple, $c_{r}(S)=\{e\}$. Therefore $Z_{G}(S) \supset R(G)$ and $S$ is normal. Then the lemma follows easily.

Proposition 4.4. Let $G$ be a Lie group such that $F(G)$ is dense in $G$. Then we have the following.

(i) $G^{0}$ has a unique maximal compact subgroup $K$; moreover $G^{0} / K$ is a central vector subgroup of $G / K$.

(ii) $G=F(G)$.

Proof. (i) Let $H=G^{0}$. Clearly $H / Z(H)$ can be identified with $\operatorname{Ad}(H) \subset$ Aut (g). By Corollary 4.2, $H / Z(H)$ has a reductive Lie algebra, hence by the preceding lemma, $H$ is the product of a connected normal semisimple Lie subgroup $S$ of $H$ and the radical $R$ of $H$. Passing over to $\bar{S}=S / S \cap R \approx H / R$, by Corollary 4.2, $\bar{S} / Z(\bar{S})$ is semisimple and compact, thus $S$ is compact. Similarly passing over to $\bar{R}=R / S \cap R \approx H / S$, by Corollary $4.2, \bar{R} / Z(\bar{R})$ is abelian and hence $R$ is nilpotent. Given $x \in \bar{R}$, the map $c_{x}: y \rightarrow x y x^{-1} y^{-1}, y \in \bar{R}$, is a homomorphism from $\bar{R}$ into $\boldsymbol{Z}(\bar{R})$. As $\boldsymbol{Z}(\bar{R})$ is a compactly generated abelian group, it has a unique maximal compact subgroup $\bar{T}$. If $x \in F(\bar{R}), c_{x}(\bar{R})$ has compact closure. Therefore $c_{x}(\bar{R}) \subset \bar{T}$ for $x \in F(\bar{R})$. Since $F(\bar{R})$ is dense in $\bar{R}$, it follows $[\bar{R}, \bar{R}] \subset \bar{T}$. Clearly $S \cap R$, contained in $Z(S)$, is a finite group. Let $T$ be the inverse image of $\bar{T}$ in $R$. It follows that $T$ is compact and $[R, R] \subset T$. Now let $K=S T^{\prime}$ where $T^{\prime}$ is the maximal compact subgroup of $R$. One sees easily that $K$ is a compact normal subgroup of $G^{0}=H$ such that $G^{0} / K$ is a vector group. The other part of the assertion is similar to that of Corollary 4.2.

(ii) From (i), $G^{0} \subset F(G)$ and hence $G=F(G) \cdot G^{0}=F(G)$.

$\left({ }^{6}\right)$ A Lie algebra $g$ is reductive if $g$ is the direct product of a semisimple Lie algebra and an abelian Lie algebra. 
THEOREM 4.5. Let $G$ be a connected locally compact group. The following are equivalent:

(i) $F(G)$ is dense in $G$.

(ii) $G=F(G)$.

(iii) $G$ has a unique maximal compact subgroup $K$ such that $G / K$ is a vector group.

Proof. (iii) $\Rightarrow$ (ii) $\Rightarrow$ (i) are trivial.

(i) $\Rightarrow$ (iii). Since $G$ is a connected locally compact group, there is a normal compact subgroup $L$ of $G$ such that $G / L$ is a Lie group. Now (i) $\Rightarrow$ (iii) follows easily from Proposition 4.4.

A topological group $G$ is called quasi-compact if $G$ is the semidirect product of a compact normal subset $K$ of $G$ and a discrete infinite cyclic subgroup $\boldsymbol{Z}$ of $G$. We are now ready to present the structure theorem of $F C$ locally compact groups.

THEOREM 4.6. Let $G$ be a locally compact group. Then G is a FC-group if and only if the following conditions are held:

(i) $G^{0}$ has a unique maximal compact subgroup $K$ of $G^{0}$.

(ii) $G^{0} / K$ is a central vector subgroup of $G / K$.

(iii) For any $x \in G$, there is a normal closed compact or quasi-compact subgroup $N$ with $x \in G^{0} N$.

Proof. ( $\Leftarrow$ ) By (i) and (ii), $G^{0} \subset F(G)$. Since any closed normal compact or quasicompact subgroup of $G$ is contained in $F(G), x \in G^{0} N \subset F(G)$.

$(\Rightarrow)$ (i) and (ii) follow from the same argument as that used in Proposition 4.4. Hence it suffices to verify (iii). We shall prove this in several cases.

Case (a). $G$ has compact-open subgroups. By Corollary $1.5, P(G)$ is a characteristic open subgroup of $G$. Hence $G / P(G)$ is a discrete $F C$-group without nontrivial periodic elements. By the same argument as that used in Proposition 1.8 (iii), $G / P(G)$ is a torsion free abelian group. Let $K$ be a compact-open subgroup of $G$, $x \in G$ and $C(G, x)=\left\{g x g^{-1}: g \in G\right\}$. Clearly there is a finite subset $\left\{x_{1}, \ldots, x_{n}\right\}$ of $C(G, x)$ with $\left\{x_{1}, \ldots, x_{n}\right\} K=K\left\{x_{1}, \ldots, x_{n}\right\}=C(G, x) K=K C(G, x)$. Since $G / P(G)$ is abelian, $x_{i}=x \lambda_{i}=\lambda_{i}^{\prime} x$ where $\lambda_{i}, \lambda_{i}^{\prime} \in P(G)(1 \leqq i \leqq n)$. By Corollary 1.5 , there is a compact normal subgroup $M$ of $G$ containing all the elements $\lambda_{i}, \lambda_{i}^{\prime}(1 \leqq i \leqq n)$. Let $K^{\prime}=M K$. By our choice of $M, C(G, x) K^{\prime}=x K^{\prime}=K^{\prime} x=K^{\prime} C(G, x)$. Clearly the subgroup $\left\langle x K^{\prime}\right\rangle$ generated by $x K^{\prime}$ is either compact or quasi-compact. Now it follows easily that the closure of $\langle C(G, x)\rangle$ is a normal closed compact or quasicompact subgroup of $G$.

Case (b). $G^{0}$ is a central vector subgroup of $G$. Let $\bar{G}=G / G^{0}, x \in G$ and $\bar{x}$ be its image in $\bar{G} .\langle C(\bar{G}, \bar{x})\rangle$ is contained in a normal closed compact or quasi-compact subgroup $\bar{L}$ of $\bar{G}$. Let $L$ be the preimage of $\bar{L}$ in $G$. If $\bar{L}$ is compact, then $L$ is the direct product of $G^{0}$ and a compact group $K\left({ }^{7}\right)$. Clearly $K$ is normal in $G$ and

( $\left.{ }^{7}\right)$ See Theorem 1, Séminaire "Sophus Lie" de L'École Normale Supérieure, 1954/55, Exposé 20, Secrétariat mathématique, Paris, 1955. 
$x \in K \cdot G^{0}$. Hence (iii) holds. If $\bar{L}$ is quasi-compact, $\bar{L}=\bar{L}_{1} A$ where $\bar{L}_{1}$ is the maximal compact-open subgroup of $\bar{L}$ and $A$ is a discrete infinite cyclic subgroup of $\bar{L}$. Let $L_{1}$ be the inverse image of $\bar{L}_{1}$ in $G$. Clearly $L_{1}=K \times G^{0}$ where $K$ is a compact group $\left({ }^{7}\right)$. Now let $y$ be any element of $G$ such that its image $\bar{y}$ in $\bar{G}$ generates $A$. $K\langle y\rangle$ is a closed quasi-compact subgroup of $G$. Since $G / K$ is a $F C$-group, it follows that for any $g \in G$,

$$
g y g^{-1} \equiv y\left(K G^{0}\right) \quad \text { iff } g y g^{-1} \equiv y(K) .
$$

Let $G_{1}=\left\{g: g y g^{-1} \equiv y\left(K G^{0}\right)\right\}$. Clearly $\left[G: G_{1}\right] \leqq 2$. If $G_{1}=G, K\langle y\rangle$ is a normal subgroup of $G$. If $G_{1} \neq G$, let $g \in G$ with $g y g^{-1} \equiv y^{-1}\left(K G^{0}\right)$. Hence

$$
g y g^{-1} \equiv y^{-1} v(K)
$$

for some $v \in G^{0}$. Let $w \in G^{0}$ with $w^{-2}=v$. Then one shows readily $g\left(y w^{\prime}\right) g^{-1}$ $\equiv(y w)^{-1}(K)$ and $h(y w) h^{-1} \equiv y w(k), h \in G_{1}$. Hence $K\langle y w\rangle$ is a quasi-compact closed normal subgroup of $G$. Clearly $x \in(K\langle y w\rangle) G^{0}$. Hence (iii) holds.

General case. Let $K$ be the maximal compact subgroup of $G^{0}$. Since by (ii) $G^{0} / K$ is a central vector subgroup of $G / K$, (iii) follows then by Case (b). Hence the proof is hereby completed.

5. Compact normal subgroups of $G$. In this section, we shall give a criterion for a subset $X$ of a locally compact group $G$ to be contained in a compact normal subgroup of $G$. First, we shall treat the following special case.

Proposition 5.1. Let $G$ be a locally compact group such that the identity component $G^{0}$ is a central vector subgroup. Let $X$ be a normal subset of $P(G)$ with compact closure. Then $X$ is contained in a compact normal subgroup of $G$.

Proof. Let $\bar{G}=G / G^{0}$ and $\bar{X}$ be the image of $X$ in $\bar{G}$. By Corollary $1.5, \bar{X}$ is contained in a compact normal subgroup $\bar{K}$ of $\bar{G}$. Let $H$ be the inverse image of $\bar{K}$ in $G$. Since $G^{0}$ is a central vector subgroup, $H$ is the direct product of compact group $K$ and $G^{0}\left({ }^{7}\right)$. Clearly $X$ is contained in $K$. As $K$ is the unique maximal compact subgroup of $H$ and $H$ is normal in $G$, it follows readily that $K$ is a compact normal subgroup of $G$.

Before we go to the general case, we shall discuss some interesting properties of Lie groups needed later. Let $G$ be a connected semisimple Lie group. It is well known that $G$ is a product of connected closed normal simple Lie subgroups $G_{i}$ $(1 \leqq i \leqq n) . G$ is called semisimple without compact factors if the factors $G_{i}(1 \leqq i \leqq n)$ are all noncompact.

Proposition 5.2 [3]. Let $G$ be a connected semisimple Lie group without compact factors and $\alpha \in$ Aut $(G)$. If the set $\left\{\alpha(g) g^{-1}: g \in G\right\}$ has compact closure, then $\alpha=e$.

Proof. Let $H=$ Aut $(G)$. Clearly $H^{0}=\operatorname{Int}(G)$ and $\left[H: H^{0}\right]$ is finite. Given $g \in G$, write $w_{g}$ for the inner automorphism $x \rightarrow g x g^{-1}, x \in G$. Clearly $\beta w_{g} \beta^{-1}=w_{\beta(g)}$, 
$g \in G, \beta \in \operatorname{Aut}(G)$. From assumption, $\left\{\alpha(g) g^{-1}: g \in G\right\}$ has compact closure, hence $\alpha \in F(H)$. Let $L$ be the identity component of the closure of $F(H)$ in $H$. From Proposition 4.4, $L$ has a unique maximal compact subgroup $K$ with abelian $L / K$. Since $H^{0}$ is semisimple without compact factors and $L$ is normal in $H^{0}$, we have $L=K=\{e\}$. It follows that $F(H)$ centralizes $H^{0}$ and the map $c_{\alpha}: g \rightarrow \alpha(g) g^{-1}, g \in G$, is a homomorphism from $G$ into $Z(G)$. Since $G$ is connected semisimple, $c_{\alpha}(G)=\{e\}$, i.e., $\alpha$ is the identity map of $G$.

5.3. Let $G$ be a connected Lie group and $R(G)$ its radical such that $[R(G), R(G)]$ is closed in $G$ and torsion free. Let $A$ be a subgroup of Aut $(G)$ such that $A$ and the subsets $\left\{\alpha(g) g^{-1}: g \in G\right\}(\alpha \in A)$ have compact closures in Aut $(G)$ and $G$ respectively.

THEOREM. Let $G$ and $A$ be described as above and $Z_{G}(A)$ be the subset $\{g: \alpha(g)=g$ for all $\alpha \in A\}$ of $G$. Then the quotient $G / Z_{G}(A)$ is compact.

Proof. We shall prove the theorem in several steps.

(i) Let $G$ be semisimple and $\beta: G \rightarrow G^{\prime}$ be a surjective homomorphism with discrete $\operatorname{ker}(\beta)$ invariant under $A$. Let $A^{\prime}$ be the image of $A$ in Aut $\left(G^{\prime}\right)$. Claim that if $G^{\prime}$ and $A^{\prime}$ satisfy the conditions of the theorem, $G / Z_{G}(A)$ is compact. Clearly $G$ is the product of a connected compact normal subgroup $K$ of $G$ and a closed connected normal semisimple Lie subgroup $H$ without compact factors. From Proposition 5.2, the restriction of $A^{\prime}$ to $\beta(H)$ is the set $\left\{1_{\beta(H)}\right\}$. Hence it follows easily the restriction of $A$ to $H$ is $\left\{1_{H}\right\}$; in particular $G / Z_{G}(A)$ is compact.

(ii) $G$ is solvable.

Since $[G, G]$ is closed in $G$ and torsion free, by induction on the length of the lower central series of $[G, G]$ one shows readily that $Z_{G}(A)$ contains $[G, G]$. Moreover if $x \in G$, and $\alpha \in A$, then $\alpha x \equiv x([G, G])$ means $\alpha x=x z$ with $z \in[G, G]$; it follows that $\alpha^{n} x=x \cdot z^{n}(n=1,2, \ldots)$. Since $\alpha \in P($ Aut $(G))$, the subset $\left\{\alpha^{n} x: n\right.$ $=1,2, \ldots\}=x \cdot\left\{z^{n}: n=1, \ldots\right\}$ has compact closure in $G$. However $[G, G]$ is closed in $G$ and torsion free, $P([G, G])=\{e\}$. It follows that $z=e$, i.e., $\alpha x \equiv x([G, G])$ iff $\alpha x=x$. Let $G^{\prime}=G /[G, G]$ and $A^{\prime}$ the image of $A$ in Aut $\left(G^{\prime}\right)$. By what we have just proved, $G / Z_{G}(A) \approx G^{\prime} \mid Z_{G^{\prime}}\left(A^{\prime}\right)$. Hence it suffices to show the assertion in case that $G$ is abelian. Since $A$ has compact closure in Aut $(G), G$ is the direct product of a compact group $K$ and a vector subgroup $V$ invariant under $A\left({ }^{8}\right)$. It follows easily $Z_{G}(A) \supset V$ and $G / Z_{G}(A)$ is compact.

(iii) General case. Since $A$ has compact closure in Aut $(G), G$ has a maximal connected semisimple Lie subgroup $S$ invariant under $A\left({ }^{9}\right)$. Clearly $G=S R(G)$, where $R(G)$ is the radical of $G$. By (ii), $R(G) / R(G) \cap Z_{G}(A)$ is compact and by (i), $S / S \cap Z_{G}(A)$ is compact. It follows then readily that $G / Z_{G}(A)$ is compact. Therefore the proof is completed.

$\left.{ }^{8}\right)$ This follows from the corresponding assertion in the Lie algebra of $G$.

( ${ }^{9}$ See [7, Corollary 5.2]. 
Lemma 5.4. Let $G$ be a connected locally compact group. Then $G$ has a maximal normal compact subgroup $K$ such that the radical $R$ of $G / K$ has the property that $[R, R]$ is closed in $G / K$ and torsion free.

Proof. It is well known that $G$ has a compact normal subgroup $L$ such that $G / L$ is a Lie group. Hence it suffices to show the lemma under the assumption that $G$ is a Lie group. Clearly by dimension argument, there is a maximal connected normal compact subgroup $M$ of $G$. Let $K^{\prime}$ be any compact normal subgroup containing $M$. Since $K^{\prime} / M$ is discrete and normal in $G / M$,

$$
K^{\prime} / M \subset Z(G / M) \text {. }
$$

$Z(G / M)$ is compactly generated, hence has a unique maximal compact subgroup $\bar{K}$ of $Z(G / M)$. Let $K$ be the preimage of $\bar{K}$ in $G$. Clearly $K$ is a maximal compact normal subgroup of $G$. Let $R$ be the radical of $G / K$ and $N$ be the maximal connected normal nilpotent subgroup of $R$. It is known that $N \supset[R, R]$ and any maximal compact subgroup of $N$ is central in $G$. Since $K$ is the maximal compact normal subgroup of $G, P(N)=\{e\}$. Clearly $N$ is closed in $G$. Now the assertions about $[R, R]$ follow immediately.

THEOREM 5.5. Let $G$ be a locally compact group and $X$ be a normal subset of $P(G)$ with compact closure. Then the group $\langle X\rangle$ generated by $X$ is a normal subgroup of $G$ with compact closure.

Proof. Let $K$ be the maximal compact normal subgroup of $G^{0}$ and $\bar{G}=G / K$. It is easy to see that without losing anything, we may assume further $G=G / K$. Hence by Lemma 5.4, in the sequel, we assume that $(*)$ : (i) $G^{0}$ is a Lie group and (ii) $\left[R\left(G^{0}\right), R\left(G^{0}\right)\right]$ is closed in $G^{0}$ and torsion free where $R\left(G^{0}\right)$ is the radical of $G^{0}$. Let $\alpha: G \rightarrow$ Aut $\left(G^{0}\right)$ be the map by assigning $g$ to the restriction on $G^{0}$ of the inner automorphism $w_{g}$. Clearly $F(\mathrm{Cl}(\alpha(F(G)))) \supset \alpha(F(G))$. Hence by Corollary 4.2 , the identity component $L$ of $\mathrm{Cl}(\alpha(F(G)))$ is the product of a compact group $M$ and a vector group $V$; moreover $L / M$ is central in $\mathrm{Cl}(\alpha(F(G))) / M$. Now by Proposition $5.1, \alpha(X)$ is contained in a compact normal subgroup of $\mathrm{Cl}(\alpha(G))$. Hence by Theorem 5.3, $G^{0} / \boldsymbol{Z}_{G^{\mathrm{o}}}(X)$ is compact. Since $X$ is normal in $G, \boldsymbol{Z}_{G^{\mathrm{o}}}(X)$ is a normal closed subgroup of $G$. Consider then the group $G / Z_{G^{0}}(X)$. By Corollary 1.5 , the group $\langle X\rangle \boldsymbol{Z}_{G^{0}}(X) / \boldsymbol{Z}_{G^{0}}(X)$ has compact closure in $G / \boldsymbol{Z}_{G^{0}}(X)$. Let $G_{1}=\boldsymbol{Z}_{G}\left(\boldsymbol{Z}_{G^{0}}(X)\right)$. Clearly $G_{1} \supset X$, and $G_{1}$ is closed normal in $G$. It is easy to see that $G_{1}^{0}$ satisfies the same assumption (*) made on $G^{0}$. Repeating the argument, we conclude $\mathrm{Cl}\left(\langle X\rangle \cdot Z_{G_{1}^{0}}(X)\right) / Z_{G_{1}^{0}}(X)$ is compact. However $Z_{G_{1}^{0}}(X) \subset Z_{G^{0}}(X)$ is central in $G_{1}$. Hence by Theorem $2.1, \mathrm{Cl}\left(\langle X\rangle \cdot Z_{G_{1}^{0}}(X)\right)$ is the direct product of a group $M$ where $M$ contains compact-open normal subgroups and a vector group $V$. Clearly $\langle X\rangle$, contained in $M$, has compact closure. Thus the theorem is hereby completed.

COROLlary 5.6. Let $G$ be a locally compact group. Then the subset $F(G) \cap P(G)$ is the characteristic subgroup of $G$ generated by all compact normal subgroups of $G$.

Proof. Immediate from Theorem 5.5. 
THEOREM 5.7. Let $G$ be a locally compact group. Then every element of $G$ is contained in a compact normal subgroup of $G$ if and only if $G=F(G)=P(G)$.

Proof. Immediate from Theorem 5.5.

6. Compactly generated groups. In this section, we shall study compactly generated topological groups and a structure theorem of such nilpotent groups is given.

LEMMA 6.1. Let $H$ be a subgroup of a locally compact group $G$. If $H$ is compactly generated, then its closure is also compactly generated.

Proof. Immediate.

THEOREM 6.2. Let $G$ be a compactly generated locally compact group, and $H$ be a closed subgroup. If $\mathrm{G} / \mathrm{H}$ is compact, then $\mathrm{H}$ is compactly generated.

Proof. Clearly there is a symmetric compact subset $K$ of $G$ such that $K$ generates $G$ and $G=K H$. Let $L=K^{3} \cap H$. Claim that $L$ generates $H$. Let $H_{0}=\langle L\rangle$. Since $G=K H, K^{2}=K^{2} \cap K H \subset K\left(K^{3} \cap H\right) \subset K H_{0}$. Hence $K^{2} H_{0} \subset K H_{0}$ and by induction $K^{n} H_{0} \subset K H_{0}$ for all positive integers $n$. Since $\langle K\rangle=G, G=K H_{0}$. It follows then that $H=H \cap K H_{0}=(H \cap K) H_{0}=H_{0}$ for $H \cap K \subset L \subset H_{0}$.

Proposition 6.3. Let $G$ be a locally compact group and $N$ be a compactly generated normal subgroup of $G$ such that $N / N \cap Z(G)$ is compact. Then $N \subset F(G)$.

Proof. Since $N / N \cap Z(G)$ is compact, $N Z(G)$ is closed and $N Z(G) / Z(G) \approx N / N$ $\cap \boldsymbol{Z}(G)$ is compact. From Corollary $2.2,[N, N]=[N \boldsymbol{Z}(G), N Z(G)]$ has compact closure in $G$. Hence we can assume that $N$ is closed in $G$ and abelian. Let $K$ be the maximal compact subgroup of $N$. Passing over to $G / K$, we may assume further that $N$ is a Lie group with $N^{0}$ isomorphic to a vector group. From the assumption, $N^{0} / N^{0} \cap \boldsymbol{Z}(G)$, isomorphic to $(N / N \cap \boldsymbol{Z}(G))^{0}$, is compact and one concludes at once $N^{0} \subset Z(G)$. Hence $N / N \cap \boldsymbol{Z}(G)$, being discrete and compact, is a finite group. Let $x \in N$ and $g \in G$. Suppose that $g x g^{-1} \equiv x(Z(G))$. Clearly $g x g^{-1} \equiv x(Z(G) \cap N)$. Now the map $x^{n} \rightarrow g x^{n} g^{-1} x^{-n}, n \in Z$, is a homomorphism from $\langle x\rangle$ into $Z(G) \cap N$. Since $N / N \cap Z(G)$ is finite and $N \cap Z(G)$ is compactly generated, $g x g \equiv x(M)$, where $M$ is the maximal compact subgroup of $N \cap Z(G)$. It follows then that $C(G, x)$ is contained in the union of finitely many cosets modulo $M$. Hence $x \in F(G)$.

The following theorem in the discrete case is due to Baer [1].

THEOREM 6.4. Let $G$ be a locally compact group and $x \in G$. Let $N(x)$ be the closure of the subgroup $\langle C(G, x)\rangle$ in $G$ and $\bar{x}$ be the image of $x$ in $G / Z(G)$. If $N(x)$ is compactly generated and $\bar{x}$ is contained in a compact normal subgroup of $G / Z(G)$, then $x \in F(G)$.

Proof. Let $H$ be the closure of $N(x) \cdot Z(G)$ in $G$. By assumption, $H / Z(G)$ is compact. Let $W$ be a compactly generated open subgroup of $H$. Clearly $N(x) W$ 
is also a compactly generated open subgroup of $H$; moreover $N(x) W \cap N(x) Z(G)$ $=N(x)(N(x) W \cap \boldsymbol{Z}(G))$ is normal in $G$. Since $N(x) Z(G)$ is dense in $H$, $N(x)(N(x) W \cap Z(G))$ is dense in $N(x) W$. It follows that $N(x) W$ is a normal subgroup of $G$. Let $N=N(x) W$. Clearly $N / N \cap Z(G)$ is compact. Hence by Proposition 6.3, the theorem follows.

In discrete case, it is well known that nilpotent groups with finite periodic generating elements are finite. In topological groups, we have the following analogy.

THOEREM 6.5. Let $G$ be a nilpotent locally compact group. If $G$ has a compact subset $K \subset P(G)$ generating a dense subgroup of $G$, then $G$ is compact.

Proof. Let $G=C^{\prime}(G) \supset \cdots \supset C^{r}(G) \supsetneqq C^{r+1}(G)=\{e\}$ be the topological lower central series, i.e., the group $C^{i+1}(G)$ is the closure of $\left[C^{i}(G), G\right]$ in $G(i=1,2, \ldots)$. If $r=1, G$ is abelian and the theorem is trivial from the structure of compactly generated abelian groups. Hence we assume that $r>1$. By induction on $r$, we assert that $G / C^{r}(G)$ and $C^{r-1}(G) / C^{r}(G)$ are compact. As $C^{r}(G)$ is central in $G, C^{r}(G)=$ the closure of $\left[G, C^{r-1}(G)\right]$ is compactly generated. Let $M$ be the maximal compact subgroup of $C^{r}(G)$. Passing over to $\bar{G}=G / M$, we have that $C^{r}(\bar{G})$ is torsion free. Let $x \in C^{r-1}(\bar{G})$. The map $c_{x}: y \rightarrow x y x^{-1} y^{-1}, y \in \bar{G}$, is a homomorphism from $\bar{G}$ into $C^{r}(\bar{G})$. Clearly $c_{x}(P(\bar{G})) \subset P\left(C^{r}(\bar{G})\right)=\{e\}$. By assumption $\langle P(\bar{G})\rangle$ is dense in $G$, hence $c_{x}(\bar{G})=\{e\}$ and $C^{r}(\bar{G})=\{e\}$. Since $M$ is compact, and by induction on $r$ $\bar{G} / C^{r}(\bar{G})=\bar{G}=G / M$ is compact, it follows easily that $G$ is compact.

7. Groups with dense $P(G)$. In this section, we want to prove the following theorem about semisimple Lie groups.

THEOREM 7.1. Let $G$ be a connected semisimple Lie group. If $P(G)$ is dense, then $G$ is compact.

Proof. Without losing anything, we may assume that $G=\operatorname{Ad}(G)$. Let $H$ be any maximal connected abelian subgroup of $G$ containing semisimple elements only. Let $G^{*}$ and $H^{*}$ be the Zarski closures of $G$ and $H$ in GL $\left(\mathrm{g} \otimes_{R} C\right)$ respectively. Then $H^{*}$ is a maximal algebraic torus of $G^{*}$. Hence the morphism $\alpha: G^{*} \times H^{*} \rightarrow G^{*}$, $\alpha((g, h))=g h g^{-1}, g \in G^{*}, h \in H^{*}$, is dominant $\left.{ }^{10}\right)$; in particular the subset $C(G, H)$ $=\bigcup_{g \in G} g \mathrm{Hg}^{-1}$ contains a nonempty open subset of $G$. Let $X$ be the subset of all nonregular elements of $G$. $X$ is a finite union of closed subsets of $G$ of dimension strictly lower than $\operatorname{dim}(G)$. Hence $C(G, H)-X$ has a nonempty open subset of $G$. Since $P(G)$ is dense, there is $t \in P(G) \cap(C(G, H)-X)$. Let $t \in\left(g \mathrm{Hg}^{-1}-X\right)$. Clearly $t$ is regular, and $H=Z_{G}\left(g^{-1} t g\right)^{0}$ is a topological torus. From this, the algebraic group $G^{*}$ is anisotropic over $R$. Hence by [2, Corollary 9.4], $G$, contained in $G_{R}^{*}$, is compact.

$\left({ }^{10}\right)$ A morphism of irreducible varieties is dominant if its image contains nonempty open subsets. 


\section{REFERENCES}

1. R. Baer, Finiteness properties of groups, Duke. Math. J. 15 (1948), 1021-1032. MR 10, 352.

2. A. Borel and J. Tits, Groupes réductifs, Inst. Hautes Études Sci. Publ. Math. No. 27 (1965), 55-150. MR 34 \#7527.

3. M. Goto, A remark on a theorem of A. Weil, Proc. Amer. Math. Soc. 20 (1969), 163-165. MR 38 \#1211.

4. S. Grosser and Martin Moskowitz, On central topological groups, Bull. Amer. Math. Soc. 72 (1966), 826-830. MR 33 \#2764.

5. S. Helgason, Differential geometry and symmetric spaces, Pure and Appl. Math., vol. 12, Academic Press, New York, 1962. MR 26 \#2986.

6. K. Iwasawa, Topological groups with invariant compact neighborhoods of the identity, Ann. of Math. (2) 54 (1951), 345-348. MR 13, 206; 1139.

7. G. D. Mostow, Fully reducible subgroups of algebraic groups, Amer. J. Math. 78 (1956), 200-221. MR 19, 1181.

8. B. H. Neumann, Groups with finite classes of conjugate elements, Proc. London Math. Soc. (3) 1 (1951), 178-187. MR 13, 316.

9. C. L. Siegel, Discontinuous groups, Ann. of Math. (2) 44 (1943), 674-689. MR 5, 228.

10. V. I. Ušakov, Classes of conjugate elements in topological groups, Ukrain. Mat. Ž. 14 (1962), 366-371. (Russian) MR 27 \#1531.

11. H.-C. Wang, On a maximality property of discrete subgroups with fundamental domain of finite measure, Amer. J. Math. 89 (1967), 124-132. MR 34 \#7708.

12. S. P. Wang, On S-subgroups of solvable groups, Amer. J. Math. 92 (1970), 389-397.

13. T. S. Wu, A certain type of locally compact totally disconnected topological groups, Proc. Amer. Math. Soc. 23 (1969), 613-614.

YALE UNIVERSITY, New Haven, Connecticut 06520 